\title{
ON THE EXTENSIONS OF VECTOR-VALUED LOEB MEASURES
}

\author{
HORST OSSWALD AND YENENG SUN
}

(Communicated by Andreas R. Blass)

\begin{abstract}
Two ways of constructing countably additive vector measures from internal vector measures are given. The connection of the extendability of vector-valued Loeb measures and the existence of the internal control measures is shown.
\end{abstract}

\section{INTRODUCTION}

Measure theory and probability theory were studied early within the context of nonstandard analysis. However, there were troublesome points, since star extensions of countably additive measures are not in general countably additive in the nonstandard universe. In 1975, Peter Loeb gave a way to construct new, rich standard measure spaces from internal measure spaces. His construction has been used in recent years to establish new standard results in many areas. Also, nonstandard analysis has proved to be a natural framework for studying local properties of Banach spaces. The central construction in that approach is the notion of nonstandard hull introduced by Luxemburg. Further material about nonstandard hulls and their applications can be found in the survey paper [3] of Henson and Moore. On the other hand, standard vector measures are central to the study of Banach space-valued functions and to the representation and classification of Banach spaces (see [1]). It is natural, therefore, to ask whether one can develop a Banach space-valued measure theory in the context of nonstandard analysis. In particular, let $\Omega$ be a nonempty internal set, $\mathscr{A}$ an internal algebra of subsets of $\Omega$, and $\nu$ a finitely additive, finite-valued internal measure with values in an internal Banach space $X$. Let $\widehat{\nu}(A)=(\nu(A))^{\wedge}$ for all $A \in \mathscr{A}$; then $\widehat{\nu}$ is a finitely additive $\widehat{X}$-valued measure. The question is, when can we find a countably additive extension of $\hat{\nu}$ to $\sigma(\mathscr{A})$. This problem was posed by Henson and Moore in 1983 (see [3, $\S 12$, Problem 17]). It is easy to see that if $X={ }^{*} \mathbb{R}$, then $\widehat{X}=\mathbb{R}$ and $\widehat{\nu}={ }^{0} \nu$. In this case, the problem is answered by Loeb's theorem in [5]. That is, $\hat{\nu}$ always has a countably additive extension to $\sigma(\mathscr{A})$. For the general case, however, this is not true, as shown

Received by the editors February 18, 1988 and, in revised form, February 5, 1990.

1980 Mathematics Subject Classification (1985 Revision). Primary 28E05; Secondary 03H05, $28 \mathrm{~B} 05$. 
by the following example:

Example. Let $X$ be the hyperfinite dimensional space $l^{\infty}(\omega)$ (see [3]), where $\omega \in \in^{*} \mathbb{N}_{\infty}$. Let $\Omega=\{i\}_{i=1}^{\omega}$ and $\mathscr{A}$ be the algebra of all internal subsets of $\Omega$. For each $i \leq \omega$, let $a_{i}=(0, \ldots, 0,1,0, \ldots, 0)$, where the 1 appears at the $i$ th place of $a_{i}$. Define $\nu(A)=\sum_{i \in A} a_{i}$ for each $A \in \mathscr{A}$. Since $\|\nu(A)\|=1$ for any nonempty internal subset of $\Omega, \nu$ is finite-valued. Thus $\nu$ is a finitely additive internal measure with finite values in $X$. If $\widehat{\nu}$ has a countably additive extension to $\sigma(\mathscr{A})$, then $\sum_{i=1}^{\infty} \widehat{\nu}(\{i\})$ is norm-convergent in $\widehat{X}$. Hence $\operatorname{Lim}_{i \rightarrow \infty} \widehat{\nu}(\{i\})=0$. But $\|\widehat{\nu}(\{i\})\|_{\widehat{X}}={ }^{0}\left(\|\nu(\{i\})\|_{X}\right)=1$ for all $i$. It follows from this contradiction that $\hat{\nu}$ has no countably additive extension to $\sigma(\mathscr{A})$.

The plan of the paper is as follows. We devote $\S 2$ to some preliminary results about standard vector measures. In $\S 3$, we solve the Henson and Moore Problem by introducing a construction of countably additive measures from internal Banach space-valued measures. In particular, we introduce the notion of internal control measures for internal Banach space-valued measures; we show that a finitely additive nonstandard hull-valued measure $\widehat{\nu}$ constructed from an internal Banach space-valued measure $\nu$ has a countably additive extension if and only if $\nu$ has an internal control measure. In $\S 4$, we present a construction of countably additive vector measures from internal, locally convex space-valued measures.

We should note that, in [7], Loeb and Osswald adopt Loeb's functional approach to nonstandard measure theory [6] to the development of integration theory in solid Riesz spaces. In [8], [9], and [10] a further study of Banach space-valued measures is presented. An integration theory of real-valued functions with respect to Banach space-valued measures is also developed. Applications include direct constructions of representing measures of both absolutely summing operators and weakly compact operators, as well as solutions of problems concerning the range of a vector measure. We also note that this paper combines the independent work of Osswald and Sun. The results in $\S 3$ can be found in [11, Chapter 2]. The results in $\S 4$ were originally obtained by Osswald. We adopt here the main framework of nonstandard analysis from [4].

\section{SOME NOTIONS AND PRELIMINARY RESULTS}

Let $X$ be a Banach space, $\Omega$ a nonempty set, $\mathscr{A}$ an algebra of subsets of $\Omega$, and $\nu$ a finitely additive set function from $\mathscr{A}$ to $X$. Let $X^{\prime}$ denote the norm dual of $X$. For each $x^{\prime} \in X^{\prime}$, let $x^{\prime}(\nu)$ denote the scalar measure defined by setting

$$
x^{\prime}(\nu)(A)=x^{\prime}(\nu(A))
$$

for each $A$ in $\mathscr{A}$, and let $\left|x^{\prime}(\nu)\right|$ denote the total variation of $x^{\prime}(\nu)$. For each $A \in \mathscr{A}$, we let

$$
\|\nu\|(A)=\sup \left\{\left|x^{\prime}(\nu)\right|(A): x^{\prime} \in X^{\prime},\left\|x^{\prime}\right\| \leq 1\right\} ;
$$


$\|\nu\|$ is called the semivariation of $\nu$. We will call $(\Omega, \mathscr{A}, \nu)$ a finitely additive bounded vector-measure space if $\|\nu\|(\Omega)$ is finite. Since only the case of bounded vector measures is considered in our study, we may just call $(\Omega, \mathscr{A}, \nu)$ a finitely additive vector-measure space. If, moreover, $\mathscr{A}$ is a $\sigma$-algebra and $\nu$ is countably additive on $\mathscr{A}$, then we will simply call $(\Omega, \mathscr{A}, \nu)$ a vectormeasure space.

Lemma 2.1. The semivariation $\|\nu\|$ is finitely subadditive and, for each $A \in \mathscr{A}$,

$$
\begin{aligned}
\sup \{\|\nu(H)\|: H \subset A, H \in \mathscr{A}\} & \leq\|\nu\|(A) \\
& \leq 2 \sup \{\|\nu(H)\|: H \subset A, H \in \mathscr{A}\} .
\end{aligned}
$$

Proof. See the proof of [1, Proposition 11, p. 4].

Definition 2.2. Let $(\Omega, \mathscr{A}, \mu)$ and $(\Omega, \mathscr{A}, \nu)$ be a finitely additive positivemeasure space and a finitely additive vector-measure space, respectively. The vector measure $\nu$ is called absolutely continuous with respect to $\mu$, or simply $\mu$-continuous, if for any positive real number $\varepsilon$ there is a positive real number $\delta$, such that for any $A \in \mathscr{A},\|\nu(A)\|<\varepsilon$ if $\mu(A)<\delta$. In this case, we say that $\mu$ is a control measure of $\nu$.

Definition 2.3. A finitely additive vector measure $\nu$ on $(\Omega, \mathscr{A})$ is said to be strongly additive if for each sequence $\left\{A_{n}\right\}$ of disjoint members of $\mathscr{A}$, the series $\sum_{n=1}^{\infty} \nu\left(A_{n}\right)$ converges in =norm. A family $\left\{\nu_{\lambda}: \lambda \in T\right\}$ of strongly additive vector measures on $(\Omega, \mathscr{A})$ is said to be uniformly strongly additive if for each sequence $\left\{A_{n}\right\}$ of pairwise disjoint members of $\mathscr{A}$,

$$
\operatorname{Lim}_{n \rightarrow \infty}\left\|\sum_{m=n}^{\infty} \nu_{\lambda}\left(A_{m}\right)\right\|=0
$$

uniformly in $\lambda \in T$.

Note. For families of countably additive measures on a $\sigma$-algebra, the concept of uniform strong additivity is precisely the familiar concept of uniform countable additivity.

Lemma 2.4. Let $\left\{\nu_{\lambda}: \lambda \in T\right\}$ be a family of countably additive vector measures on the measure space $(\Omega, \sigma(\mathscr{A}))$. Then $\left\{\nu_{\lambda}: \lambda \in T\right\}$ is uniformly countably additive on $\sigma(\mathscr{A})$ if and only if the family of restrictions $\left\{\left.\nu_{\lambda}\right|_{\mathscr{\infty}}: \lambda \in T\right\}$ is uniformly strongly additive on $\mathscr{A}$.

Proof. See [1, Lemma 1, p. 26].

Lemma 2.5. Let $\Gamma=\left\{\mu_{i}: i \in I\right\}$ be a set of uniformly countably additive scalar measures on the measure space $(\Omega, \sigma(\mathscr{A}))$. Then for any positive real number $\varepsilon$, there are elements $\mu_{1}, \mu_{2}, \ldots, \mu_{n}$ of $\Gamma$ such that for any $B \in \sigma(\mathscr{A})$, if $\mu_{i}(B)=0$ for $1 \leq i \leq n$, then

$$
\sup \left\{\mu_{i}(B): i \in I\right\}<\varepsilon .
$$

Proof. See the proof in [1, p. 12]. 


\section{The CONSTRUCTION OF LOEB MEASURES IN A NONSTANDARD HULL}

Let $X$ be an internal Banach space in some $\aleph_{1}$-saturated nonstandard model, $\Omega$ a nonempty internal set, $\mathscr{A}$ an internal algebra of subsets of $\Omega$, and $\nu$ an internal, finitely additive set function from $\mathscr{A}$ to $X$. By transferring the definition of the semivariation of a standard vector measure, we can define the internal semivariation of $\nu$, denoted by $\|\nu\|$. Thus, as in $\S 2$, we call $\nu$ a bounded, finitely additive internal vector measure and $(\Omega, \mathscr{A}, \nu)$ a bounded finitely additive internal vector-measure space, if $\|\nu\|(\Omega)$ is a finite hyperreal number. Since only finite additivity and the case of bounded vector measures are concerned for internal measures, we use the term "internal vector measures" instead of "bounded finitely additive internal vector measures." We may also simply call $(\Omega, \mathscr{A}, \nu)$ an internal vector-measure space.

Definition 3.1. A bounded, internal, hyperreal-valued positive measure $\mu$ on $(\Omega, \mathscr{A})$ is said to be an internal control measure of $\nu$ if for all $A$ with $\mu(A) \approx$ 0 we have $\nu(A)$ in the monad of 0 in $X$.

As usual, $\widehat{X}$ denotes the norm nonstandard hull of $X$. We define a set function $\widehat{\nu}$ from $\mathscr{A}$ to $\widehat{X}$ by setting $\widehat{\nu}(A)=(\nu(A))^{\wedge}$ for each $A \in \mathscr{A}$. It is obvious that $\hat{\nu}$ is finitely additive. Let $\sigma(\mathscr{A})$ be the $\sigma$-algebra generated by $\mathscr{A}$. We now state the main theorem of this section:

Theorem 3.2. $\widehat{\nu}$ has a countably additive extension to $\sigma(\mathscr{A})$ if and only if $\nu$ has an internal control measure.

Note. Rade Zivaljevic showed in [12] that $\widehat{\nu}$ has a countably additive extension to $\sigma(\mathscr{A})$ if $\nu$ is of bounded variation. His result is a special case of the sufficiency part of our theorem, since we can take the internal variation $|\nu|$ of $\nu$ as an internal control measure of $\nu$. The following example can be found in [8, $\S 2]$. It shows the existence of such vector measures, which have internal control measures but are not of bounded variation. Therefore, the sufficiency part of our theorem yields extensions for measures not covered by Rade Zivaljevic's result.

Example 3.3. Let $X$ be the hyperfinite dimensional space $l^{2}(\omega)$ (see [3]), where $\omega$ is infinite in ${ }^{*} \mathbb{N}$. Let $\Omega=\{i\}_{i=1}^{\omega}$, and let $\mathscr{A}$ be the algebra of all internal subsets of $\Omega$. For each $i \leq \omega$, let $a_{i}=(0, \ldots, 0,1 / \sqrt{\omega}, 0, \ldots, 0)$, where the $1 / \sqrt{\omega}$ appears at the $i$ th place of $a_{i}$. Define $\nu(A)=\sum_{i \in A} a_{i}$ for each $A \in \mathscr{A}$. Let $\mu$ be the counting probability measure on $\Omega$. Then $\mu$ is an internal control measure of the bounded vector measure $\nu$, but $\nu$ is not of bounded variation.

Now we are going to prove several lemmas which are needed in the proof of the main theorem.

Lemma 3.4. A bounded internal measure $\mu$ is an internal control measure of $\nu$ if and only if, for any positive real number $\varepsilon$, there is a positive real number $\delta$ such that for any $A \in \mathscr{A},\|\nu(A)\|<\varepsilon$ if $\mu(A)<\delta$. 
Proof. Assume $\mu$ is an internal control measure of $\nu$ and $\varepsilon$ is an arbitrary positive real number. Fix $n \in \in^{*} \mathbb{N}_{\infty}$. If $A$ is some element in the internal algebra $\mathscr{A}$ with $\mu(A)<1 / n$, then $\mu(A) \approx 0$, so

$$
\nu(A) \approx 0, \quad \text { which implies that }\|\nu(A)\|<\varepsilon .
$$

The sentence $\alpha(n)=$ " $(\forall A \in \mathscr{A})[\mu(A)<1 / n \Rightarrow\|\nu(A)\|<\varepsilon]$ " is an internal one, so by the Permanence Principle, $\alpha(n)$ holds for some standard positive integer $m$. We set $\delta$ equal to $1 / m$.

To prove the converse, fix $A \in \mathscr{A}$ with $\mu(A) \approx 0$, and for a given positive real number $\varepsilon$, let $\delta$ be the corresponding positive real number in the statement of the lemma. We have $\mu(A)<\delta$; hence

$$
\|\nu(A)\|<\varepsilon .
$$

Since $\varepsilon$ is arbitrary, we have $\nu(A) \approx 0$.

Lemma 3.5. For each $A \in \mathscr{A}$,

$$
\begin{aligned}
\sup \{\|\nu(H)\|: H \subset A, H \in \mathscr{A}\} & \leq\|\nu\|(A) \\
& \leq 2 \sup \{\|\nu(H)\|: H \subset A, H \in \mathscr{A}\} .
\end{aligned}
$$

Proof. Transfer of Lemma 2.1.

Lemma 3.6. If $(\Omega, \mathscr{A}, m)$ is a bounded internal scalar-measure space, then

$$
{ }^{0}|m|=\left|{ }^{0} m\right| \text { on }(\Omega, \mathscr{A}) \text {, }
$$

where ${ }^{0}|m|$ and ${ }^{0} m$ are defined on $(\Omega, \mathscr{A})$ by ${ }^{0}|m|(A)=\operatorname{st}(|m|(A))$ and ${ }^{0} m(A)=\operatorname{st}(m(A))$, for all $A \in \mathscr{A}$.

Proof. Choose any $E \in \mathscr{A}$ and pick any finite partition $\pi$ of $E$. Then

$$
\left.\sum_{A \in \pi}\right|^{0} m(A)\left|=\sum_{A \in \pi}^{0}\right| m(A)\left|\leq^{0}\right| m \mid(E),
$$

so $\left.\right|^{0} m\left|(E) \leq^{0}\right| m \mid(E)$ follows from the arbitrary choice of $\pi$.

It remains to prove that $\left.\right|^{0} m\left|(E) \geq^{0}\right| m \mid(E)$ for all $E \in \mathscr{A}$. If not, $\exists \varepsilon \in \mathbb{R}^{+}$ and $E \in \mathscr{A}$ such that ${ }^{0}|m|(E)>\left.\right|^{0} m \mid(E)+\varepsilon$; thus, $|m|(E)>\left.\right|^{0} m \mid(E)+\varepsilon$. Hence, there exists a hyperfinite partition $\pi=\left\{A_{i}\right\}_{i=1}^{\omega}$ of $E$ such that

$$
\sum_{i=1}^{\omega}\left|m\left(A_{i}\right)\right|>\left.\right|^{0} m \mid(E)+\varepsilon
$$

Let

$$
A^{\prime}=\bigcup_{m\left(A_{i}\right) \geq 0} A_{i} \text { and } A^{\prime \prime}=\bigcup_{m\left(A_{i}\right)<0} A_{i}
$$

then

$$
m\left(A^{\prime}\right)-m\left(A^{\prime \prime}\right)>\left.\right|^{0} m \mid(E)+\varepsilon .
$$

Hence

$$
{ }^{0} m\left(A^{\prime}\right)-{ }^{0} m\left(A^{\prime \prime}\right) \geq\left|{ }^{0} m\right|(E)+\varepsilon \text {. }
$$


Therefore $\left|{ }^{0} m\right|(E)+\varepsilon \leq\left.\right|^{0} m\left(A^{\prime}\right)|+|^{0} m\left(A^{\prime \prime}\right)|\leq|^{0} m \mid(E)$. This is a contradiction.

Lemma 3.7. If $\hat{\nu}$ has a countably additive extension to $\sigma(\mathscr{A})$, then for $\left\{A_{n}\right\} a$ sequence of disjoint members of $\mathscr{A}$, we have

$$
\operatorname{Lim}_{n \rightarrow \infty}{ }^{0}\|\nu\|\left(A_{n}\right)=0 .
$$

Proof. Suppose not: then $\exists \varepsilon_{0} \in \mathbb{R}^{+}$and a subsequence $\left\{A_{n_{k}}\right\}$ of $\left\{A_{n}\right\}$ such that ${ }^{0}\|\nu\|\left(A_{n_{k}}\right)>2 \varepsilon_{0}$, whence $\|\nu\|\left(A_{n_{k}}\right)>2 \varepsilon_{0}$. Therefore,

$$
\sup \left\{\|\nu(H)\|: H \subset A_{n_{k}}, H \in \mathscr{A}\right\}>\varepsilon_{0}
$$

by Lemma 3.5. Thus $\exists H_{n_{k}} \subset A_{n_{k}}$ with $H_{n_{k}} \in \mathscr{A}$ such that $\left\|\nu\left(H_{n_{k}}\right)\right\|>\varepsilon_{0}$, whence $\left\|\widehat{\nu}\left(H_{n_{k}}\right)\right\| \geq \varepsilon_{0}$ for all $k \in \mathbb{N}$. We now have a contradiction with the countable extendability of the bounded measure $\widehat{\nu}$.

Lemma 3.8. If $\widehat{\nu}$ has a countably additive extension to $\sigma(\mathscr{A})$, then the family $\left\{\left.\right|^{0} x^{\prime}(\nu) \mid: x^{\prime} \in X^{\prime},\left\|x^{\prime}\right\| \leq 1\right\}$ is uniformly strongly additive on $\mathscr{A}$.

Proof. Suppose not; then there is a sequence $\left\{A_{n}\right\}$ of disjoint sets in $\mathscr{A}$ and an $\varepsilon \in \mathbb{R}^{+}$such that

$$
\sup \left\{\left.\sum_{k=n}^{\infty}\right|^{0} x^{\prime}(\nu) \mid\left(A_{k}\right): x^{\prime} \in X^{\prime},\left\|x^{\prime}\right\| \leq 1\right\}>2 \varepsilon
$$

for all $n \in \mathbb{N}$, wilence there exists an increasing sequence of positive integers $\left\{n_{j}\right\}$ such that

$$
\sup \left\{\left.\sum_{k=n_{j}+1}^{n_{j+1}}\right|^{0} x^{\prime}(\nu) \mid\left(A_{k}\right): x^{\prime} \in X^{\prime},\left\|x^{\prime}\right\| \leq 1\right\}>\varepsilon
$$

i.e.,

$$
\sup \left\{\left.\right|^{0} x^{\prime}(\nu) \mid\left(\sum_{k=n_{j}+1}^{n_{j+1}} A_{k}\right): x^{\prime} \in X^{\prime},\left\|x^{\prime}\right\| \leq 1\right\}>\varepsilon
$$

for all $j$. Let

$$
H_{j}=\bigcup_{k=n_{j}+1}^{n_{j+1}} A_{k}
$$

Then $\left\{H_{j}\right\}$ is a sequence of disjoint sets in $\mathscr{A}$. For each $j \in \mathbb{N}$,

$$
\sup \left\{{ }^{0}\left|x^{\prime}(\nu)\right|\left(H_{j}\right): x^{\prime} \in X^{\prime},\left\|x^{\prime}\right\| \leq 1\right\}<\varepsilon,
$$

by Lemma 3.6. Hence, for each $j \in \mathbb{N}$,

$$
\|\nu\|\left(H_{j}\right)=\sup \left\{\left|x^{\prime}(\nu)\right|\left(H_{j}\right): x^{\prime} \in X^{\prime},\left\|x^{\prime}\right\| \leq 1\right\}>\varepsilon,
$$

which contradicts Lemma 3.7. 
Lemma 3.9. If $\hat{\nu}$ has a countably additive extension to $\sigma(\mathscr{A})$, then $\left\{L\left(\left|x^{\prime}(\nu)\right|\right)\right.$ : $\left.x^{\prime} \in X^{\prime},\left\|x^{\prime}\right\| \leq 1\right\}$ is uniformly countably additive on $\sigma(\mathscr{A})$, where $L\left(\left|x^{\prime}(\nu)\right|\right)$ is the scalar Loeb measure on $\sigma(\mathscr{A})$ derived from $\left|x^{\prime}(\nu)\right|$.

Proof. Use Lemmas 2.4 and 3.8.

Assume now that $\mu$ is an internal control measure of $\nu$ and $(\Omega, \sigma(\mathscr{A})$, $L(\mu))$ is the corresponding Loeb space. We know that for any $B \in \sigma(\mathscr{A})$, $\exists A \in \mathscr{A}$ such that $L(\mu)(A \Delta B)=0$. We therefore can define a set function on $\sigma(\mathscr{A})$ with values in $\widehat{X}$.

Definition 3.10. For each $B \in \sigma(\mathscr{A})$, set $L(\nu)(B)=\widehat{\nu}(A)$, where $A$ is chosen from $\mathscr{A}$ so that $L(\mu)(A \Delta B)=0$.

Proposition 3.11. $L(\nu)$ is a well-defined, finitely additive set function on $\sigma(\mathscr{A})$. Proof. First we check that $L(\nu)$ is well defined. Fix $B \in \sigma(\mathscr{A})$ and $A_{1}$, $A_{2} \in \mathscr{A}$ such that $L(\mu)\left(A_{1} \Delta B\right)=L(\mu)\left(A_{2} \Delta B\right)=0$. Then $A_{1} \Delta A_{2} \subset\left(A_{1} \Delta B\right) \cup$ $\left(A_{2} \Delta B\right)$, so $L(\mu)\left(A_{1} \Delta A_{2}\right)=0, \mu\left(A_{1}-A_{2}\right) \approx 0$. Similarly $\mu\left(A_{2}-A_{1}\right) \approx 0$. Thus $\nu\left(A_{1}-A_{2}\right) \approx 0, \nu\left(A_{2}-A_{1}\right) \approx 0$. Hence

$$
\begin{aligned}
\nu\left(A_{1}\right) & =\nu\left(A_{1}-A_{2}\right)+\nu\left(A_{1} \cap A_{2}\right) \\
& \approx \nu\left(A_{1} \cap A_{2}\right) \\
& \approx \nu\left(A_{2}-A_{1}\right)+\nu\left(A_{1} \cap A_{2}\right)=\nu\left(A_{2}\right),
\end{aligned}
$$

which implies that $\widehat{\nu}\left(A_{1}\right)=\widehat{\nu}\left(A_{2}\right)$. Therefore $L(\nu)$ is well defined. It is easy to check that $L(\nu)$ is finitely additive.

Now we are ready to present a proof for the main theorem.

Proof of the "sufficiency" part of Theorem 3.2. Let $\mu$ be an internal control measure of $\nu$, and let $L(\nu)$ be the mapping from $\sigma(\mathscr{A})$ to $\hat{X}$ given by Definition 3.10. We will prove that $L(\nu)$ is a countably additive extension of $\hat{\nu}$ to $\sigma(\mathscr{A})$. It is clear that $L(\nu)$ is an extension of $\widehat{\nu}$. By Lemma 3.4, for any positive real number $\varepsilon$ there is a positive real number $\delta$ such that, for any $A \in \mathscr{A},\|\nu(A)\|<\varepsilon$ if $\mu(A)<\delta$. It follows from the definition of $L(\nu)$ that, for any $B \in \sigma(\mathscr{A})$, we have $\|L(\nu)(B)\|<\varepsilon$ if $L(\mu)(B)<\delta$. Given an increasing sequence $\left\{B_{n}\right\}$ in $\sigma(\mathscr{A})$, let $\bigcup_{n=1}^{\infty} B_{n}=B$. Since $L(\mu)$ is countably additive by Loeb's theorem, we have

$$
\operatorname{Lim}_{n \rightarrow \infty} L(\mu)\left(B_{n}\right)=L(\mu)(B) .
$$

Thus, there is an $N_{0} \in \mathbb{N}$ such that, for $n>N_{0}, L(\mu)\left(B-B_{n}\right)<\delta$, whence $\left\|L(\nu)\left(B-B_{n}\right)\right\|<\varepsilon$; i.e., $\left\|L(\nu)(B)-L(\nu)\left(B_{n}\right)\right\|<\varepsilon$. Therefore

$$
\operatorname{Lim}_{n \rightarrow \infty} L(\nu)\left(B_{n}\right)=L(\nu)(B),
$$

which means that $L(\nu)$ is countably additive.

Proof of the "necessity" part of Theorem 3.2. Assume $\hat{\nu}$ has a countably additive extension to $\sigma(\mathscr{A})$. By Lemmas 2.5 and 3.9, there exist for any $\varepsilon \in \mathbb{R}^{+}$ 
functionals $\left(x^{\prime}\right)_{1}^{\varepsilon}, \ldots,\left(x^{\prime}\right)_{1}^{\varepsilon} \in X^{\prime}$ with $\left\|\left(x^{\prime}\right)_{i}^{\varepsilon}\right\| \leq 1$ for $1 \leq i \leq n$, such that for any $B \in \sigma(\mathscr{A})$, if $\left.L\left(\mid C x^{\prime}\right)_{i}^{\varepsilon}(\nu) \mid\right)(B)=0$ for $1 \leq i \leq n$, then

$$
\sup \left\{L\left(x^{\prime}(\nu) \mid\right)(B): x^{\prime} \in X^{\prime},\left\|x^{\prime}\right\| \leq 1\right\}<\varepsilon .
$$

For each $\varepsilon=1 / m, m \in \mathbb{N}$, we get a finite sequence of such internal linear functionals. We put all of them together to get an infinite sequence of internal linear functionals $\left\{x_{n}^{\prime}\right\}_{n=1}^{\infty}$ with $x_{n}^{\prime} \in X^{\prime}$ and $\left\|x_{n}^{\prime}\right\| \leq 1$ such that for any $B \in \sigma(\mathscr{A})$ and any $x^{\prime} \in X^{\prime}$ with $\left\|x^{\prime}\right\| \leq 1$, if $L\left(\left|x_{n}^{\prime}(\nu)\right|\right)(B)=0$ for all $n \in \mathbb{N}$, then $L\left(\left|x^{\prime}(\nu)\right|\right)(B)=0$. By $\aleph_{1}$-saturation there is an internal extension $\left\{x_{n}^{\prime}: n \in^{*} \mathbb{N}\right\}$ of $\left\{x_{n}^{\prime}\right\}$ to the unit ball of $X^{\prime}$. Let $\omega$ be a positive infinite integer; we define

$$
\mu(A)=\sum_{n=1}^{\infty} \frac{1}{2^{n}}\left|x_{n}^{\prime}(\nu)\right|(A) \quad \text { for all } A \in \mathscr{A} .
$$

Now $\mu$ is an internal and finitely additive set function from $\mathscr{A}$ to ${ }^{*} \mathbb{R}^{+}$. For each $A \in \mathscr{A}, \mu(A) \leq \sum_{n=1}^{\omega} \frac{1}{2^{n}}\|\nu\|(A) \leq\|\nu\|(A)$. Fix $A \in \mathscr{A}$ so that $\mu(A) \approx 0$. Then for all $n \in N,\left|x_{n}^{\prime}(\nu)\right|(A) \approx 0$, which implies that

$$
L\left(\left|x_{n}^{\prime}(\nu)\right|\right)(A)=0 .
$$

In this case, for all $x^{\prime} \in X^{\prime}$ with $\left\|x^{\prime}\right\| \leq 1, L\left(\left|x^{\prime}(\nu)\right|\right)(A)=0$; that is, $\left|x^{\prime}(\nu)\right|(A) \approx 0$. By the definition of $\|\nu\|$, we have $\|\nu\|(A) \approx 0$; hence $\nu(A) \approx 0$ by Lemma 3.5. We also know that $\mu(\Omega)$ is a finite number, since

$$
\mu(\Omega) \leq\|\nu\|(\Omega) .
$$

Therefore, $\mu$ is an internal control measure of $\nu$.

Corollary 3.12. If $\widehat{\nu}$ has a countably additive extension to $\sigma(\mathscr{A})$ (which is necessarily unique), then we can find an internal control measure $\mu$ of $\nu$ with

$$
0 \leq \mu(A) \leq\|\nu\|(A) \text { for all } A \in \mathscr{A} .
$$

Theorem 3.13. The range of $L(\nu)$ on $(\Omega, \sigma(\mathscr{A}))$ is $(\nu(\mathscr{A}))^{\wedge}$, which is closed in $\widehat{X}$.

Proof. It follows from the definition of $L(\nu)$ that its range is $(\nu(\mathscr{A}))^{\wedge}$. Let $\left\{y_{n}\right\}$ be a sequence of elements in the range of $L(\nu)$ with $\operatorname{Lim}_{n \rightarrow \infty} y_{n}=y_{0}$ in $\widehat{X}$. For each $n \in \mathbb{N}, \exists B_{n} \in \sigma(\mathscr{A})$ such that $L(\nu)\left(B_{n}\right)=y_{n}$, and by the definition of $L(\nu), \exists A_{n} \in \mathscr{A}$ such that $L(\nu)\left(B_{n}\right)=\widehat{\nu}\left(A_{n}\right)$. Choose an internal extension $\left\{A_{n}: n \in \in^{*} \mathbb{N}\right\}$ of $\left\{A_{n}\right\}$. Since $\left\{y_{n}\right\}$ is Cauchy in $\widehat{X}$, for each $k \in \mathbb{N}$, $\exists n_{k} \in \mathbb{N}$ such that for all $n, m \in \mathbb{N}$ with $n \geq n_{k}$ and $m \geq n_{k}$,

$$
\left\|\nu\left(A_{n}\right)-\nu\left(A_{m}\right)\right\|<\frac{1}{k} \text {. }
$$

By the Permanence Principle, $\exists N_{k} \in \in^{*} \mathbb{N}_{\infty}$ such that $\left\|\nu\left(A_{n}\right)-\nu\left(A_{m}\right)\right\|<\frac{1}{k}$ for all $n, m \in{ }^{*} \mathbb{N}$ with $n_{k} \leq n, m \leq N_{k}$. By $\aleph_{1}$-saturation, there is an $N_{0} \in \mathbb{N}_{\infty}$ 
such that $N_{0} \leq N_{k}$ for all $k \in \mathbb{N}$. Now $\left\|\nu\left(A_{n}\right)-\nu\left(A_{N_{0}}\right)\right\|<\frac{1}{k}$ for all $n \in \mathbb{N}$ with $n \geq n_{k}$. Therefore $\widehat{\nu}\left(A_{N_{0}}\right)$ is the limit of $\left\{y_{n}\right\}$, so

$$
y_{0}=\widehat{\nu}\left(A_{N_{0}}\right)=L(\nu)\left(A_{N_{0}}\right) \text {. }
$$

It follows from the arbitrary choice of the sequence that the range of $L(\nu)$ is closed.

\section{LOCALly CONVEX SPACE-VALUED LOEB MEASURES}

Let $X$ be a locally convex topological vector space, $X^{\prime}$ the topological dual of $X, \Omega$ an internal nonempty set, $\mathscr{A}$ an internal algebra of subsets of $\Omega$, $\nu$ a finitely additive ${ }^{*} X$-valued internal set function on $\mathscr{A}$, and $\kappa$ a cardinal number greater than the cardinality of $X^{\prime}$. We assume the nonstandard model is $\kappa$-saturated and each point in the range of $\nu$ is near-standard in the weak topology of $X$. Then we can define a finitely additive $X$-valued measure ${ }^{0} \nu$ on $(\Omega, \mathscr{A})$ by taking the standard part of $\nu$ in the weak topology of $X$. That is, ${ }^{0} \nu(A)={ }^{0}(\nu(A))_{w}$ for each $A \in \mathscr{A}$. Now we may ask if ${ }^{0} \nu$ has a countably additive extension to $\sigma(\mathscr{A})$. We will answer this question by presenting a new construction of vector Loeb measures from the internal measure $\nu$.

For any linear functional $\varphi \in X^{\prime}$, let

$$
{ }^{*} \varphi(\nu)(A)={ }^{*} \varphi(\nu(A)) \text { and } \varphi\left({ }^{0} \nu\right)(A)=\varphi\left({ }^{0} \nu(A)\right),
$$

for each $A \in \mathscr{A}$. We know that

$$
{ }^{*} \varphi(\nu)(A)=\varphi\left({ }^{0} \nu(A)\right) \approx{ }^{*} \varphi(\nu(A))={ }^{*} \varphi(\nu)(A),
$$

for each $A \in \mathscr{A}$. So $\varphi\left({ }^{0} \nu\right)$ and ${ }^{0}\left({ }^{*} \varphi(\nu)\right)$ are the same measures on $(\Omega, \mathscr{A})$. We will use $L\left({ }^{*} \varphi(\nu)\right)$ to denote the Loeb measure and $L_{\varphi}(\mathscr{A})$ to denote the corresponding Loeb algebra. Then $\left(\Omega, L_{\varphi}(\mathscr{A}), L\left({ }^{*} \varphi(\nu)\right)\right)$ is a complete measure space.

Definition 4.1. A subset $B$ of $\Omega$ is called $\nu$-measurable if we can find $A \in \mathscr{A}$ such that, for every $\varphi \in X^{\prime}, A \Delta B \in L_{\varphi}(\mathscr{A})$ and $\left|L\left(^{*} \varphi(\nu)\right)\right|(A \Delta B)=0$. The set $A$ is called a $\nu$-approximation of $B$. We use $L_{\nu}(\mathscr{A})$ to denote the class of all $\nu$-measurable sets.

Definition 4.2. For each $B \in L_{\nu}(\mathscr{A})$, set

$$
L(\nu)(B)={ }^{0} \nu(A),
$$

where $A$ is chosen from $\mathscr{A}$ such that $A$ is a $\nu$-approximation of $B$.

Proposition 4.3. $L_{\nu}(\mathscr{A})$ is a $\sigma$-algebra and $L(\nu)$ is a well-defined, countably additive measure in the original topology of $X$.

Proof. It is easy to check that $L_{\nu}(\mathscr{A})$ is an algebra and $L(\nu)$ is a well-defined finitely additive set function on $L_{\nu}(\mathscr{A})$. To prove $L_{\nu}(\mathscr{A})$ is a $\sigma$-algebra, 
we choose any increasing sequence $\left\{B_{n}\right\}$ in $L_{\nu}(\mathscr{A})$; then we can find $\nu$ approximations $A_{1}, A_{2}, \ldots, A_{n}, \ldots$ of $B_{1}, B_{2}, \ldots, B_{n}, \ldots$ such that $\left\{A_{n}\right\}$ is an increasing sequence. For each $\varphi \in X^{\prime}$, let

$$
S_{\varphi}=\sup _{n \in \mathbb{N}} 0^{*} \varphi(\nu) \mid\left(A_{n}\right) .
$$

Since the nonstandard model is $\kappa$-saturated, there exists $A \in \mathscr{A}$ such that for all $n \in \mathbb{N}, A_{n} \subset A$ and for all $\varphi \in X^{\prime}, m \in \mathbb{N}, I^{*} \varphi(\nu) \mid(A) \leq S_{\varphi}+\frac{1}{m}$.

Claim. $A$ is a $\nu$-approximation of $\bigcup_{n=1}^{\infty} A_{n}$.

We know that, for each $\varphi \in X^{\prime}, \bigcup_{n=1}^{\infty} A_{n} \in \sigma(\mathscr{A}) \subset L_{\varphi}(\mathscr{A})$. Thus, for each $k$ and $m \in \mathbb{N}$,

$$
\begin{aligned}
L\left(^{*} \varphi(\nu)\right) \mid\left(A-\bigcup_{n=1}^{\infty} A_{n}\right) & \leq\left|L\left({ }^{*} \varphi(\nu)\right)\right|\left(A-A_{k}\right)=L\left(\left.\right|^{*} \varphi(\nu) \mid\right)\left(A-A_{k}\right) \\
& =\left.{ }^{0}\right|^{*} \varphi(\nu)\left|(A)-{ }^{0}\right|^{*} \varphi(\nu)\left|\left(A_{k}\right) \leq S_{\varphi}+\frac{1}{m}-{ }^{0}\right|^{*} \varphi(\nu) \mid\left(A_{k}\right) .
\end{aligned}
$$

Therefore, $\left|L\left({ }^{*} \varphi(\nu)\right)\right|\left(A-\bigcup_{n=1}^{\infty} A_{n}\right)=0$. Thus the claim follows from the arbitrary choice of $\varphi$.

It is clear that $A \Delta \bigcup_{n=1}^{\infty} B_{n} \subset \bigcup_{n=1}^{\infty}\left(A_{n} \Delta B_{n}\right) \cup\left(A \Delta \bigcup_{n=1}^{\infty} A_{n}\right)$. Thus, $\left|L\left({ }^{*} \varphi(\nu)\right)\right|\left(A \Delta \bigcup_{n=1}^{\infty} B_{n}\right)=0$ for each $\varphi \in X^{\prime}$. So $\bigcup_{n=1}^{\infty} B_{n}$ is $\nu$-measurable. Therefore, $L_{\nu}(\mathscr{A})$ is a $\sigma$-algebra. Moreover, for each $\varphi \in X^{\prime}$,

$$
\begin{aligned}
\varphi(L(\nu))\left(\bigcup_{n=1}^{\infty} B_{n}\right) & =\varphi\left(L(\nu)\left(\bigcup_{n=1}^{\infty} B_{n}\right)\right)=\varphi\left({ }^{0} \nu(A)\right)={ }^{0}\left(\varphi\left(^{*}(\nu)\right)(A)\right. \\
& =L\left(^{*} \varphi(\nu)\right)(A) \\
& =L\left(^{*} \varphi(\nu)\right)\left(A-\bigcup_{n=1}^{\infty} A_{n}\right)+L\left(^{*} \varphi(\nu)\right)\left(\bigcup_{n=1}^{\infty} A_{n}\right) \\
& =L\left(^{*} \varphi(\nu)\right)\left(\bigcup_{n=1}^{\infty} A_{n}\right) \\
& =\operatorname{Lim}_{n \rightarrow \infty} L\left(^{*} \varphi(\nu)\right)\left(A_{n}\right)=\operatorname{Lim}_{n \rightarrow \infty}{ }^{0}\left(^{*} \varphi(\nu)\right)\left(A_{n}\right)=\operatorname{Lim}_{n \rightarrow \infty} \varphi\left({ }^{0} \nu\right)\left(A_{n}\right) \\
& =\operatorname{Lim}_{n \rightarrow \infty} \varphi\left(L(\nu)\left(B_{n}\right)\right)=\operatorname{Lim}_{n \rightarrow \infty} \varphi(L(\nu))\left(B_{n}\right) .
\end{aligned}
$$

Hence, $L(\nu)$ is weakly countably additive. The countable additivity of $L(\nu)$ in the original topology follows from a result of Grothendieck [2], which says that every weakly countably additive measure with values in a locally convex topological space is also countably additive in the original topology of the space.

It is known that the locally convex topology of $X$ can be generated by a family $\left\{\|\cdot\|_{\rho}\right\}_{\rho<\alpha}$ of seminorms. For a linear functional $\varphi \in X^{\prime}$, we say $\|\varphi\|_{\rho} \leq 1$ if $|\varphi(x)| \leq\|x\|_{\rho}$, for all $x \in X$. In $\S 3$, we constructed internal control measures of the internal Banach space-valued measures. It is natural to ask whether it is possible to construct internal control measures for the locally 
convex space-valued measures studied in this section in the same sense. It will be seen that we can give similar constructions by adding some conditions on $L(\nu)$.

Definition 4.4. The Loeb measure $L(\nu)$ is called $\sigma$-separating if there exists a sequence $\left\{\|\cdot\|_{n}\right\}$ in $\left\{\|\cdot\|_{\rho}\right\}_{\rho<\alpha}$ such that, for all $B \in L_{\nu}(\mathscr{A}), L(\nu)(B) \neq 0$ implies that there exists $n \in \mathbb{N}$ with $\|L(\nu)(B)\|_{n} \neq 0$.

It should be noted that $L(\nu)$ is $\sigma$-separating if $X$ is metrizable or one of the seminorms is a norm. Now we suppose $L(\nu)$ is $\sigma$-separating and let $\left\{\|\cdot\|_{n}\right\}$ be a countable sequence of seminorms separating $L(\nu)$. We call a functional $\varphi \in X^{\prime}$ normal if $\left.|\varphi(L(\nu))|(\Omega)={ }^{0}\left(\left.\right|^{*} \varphi(\nu) \mid\right)(\Omega)\right)<1$.

Lemma 4.5. For any given $B \in L_{\nu}(\mathscr{A})$, if $|\varphi(L(\nu))|(B)=0$ for all normal continuous linear functionals $\varphi$ with the property $\|\varphi\|_{n} \leq 1$ for some $n \in \mathbb{N}$, then, for all $\varphi \in X^{\prime},|\varphi(L(\nu))|(B)=0$.

Proof. Suppose there exists some $\varphi$ in $X^{\prime}$ such that $|\varphi(L(\nu))|(B) \neq 0$. Then there is a $C$ in $L_{\nu}(\mathscr{A})$ such that $C \subset B$ and $\varphi(L(\nu))(C) \neq 0$. Thus, $L(\nu)(C) \neq 0$. Since $L(v)$ is $\sigma$-separating, $\exists n \in \mathbb{N}$. $\|L(\nu)(C)\|_{n} \neq 0$. By the Hahn-Banach Theorem, there exists a normal continuous linear functional $\psi$ such that $\|\psi\|_{n} \leq 1$ and $\psi(L(\nu))(C) \neq 0$. Therefore $|\psi(L(\nu))|(B) \geq$ $|\psi(L(\nu))|(C)>0$. That contradicts the hypothesis of the lemma.

Lemma 4.6. Fix $n \in \mathbb{N}$. There exists a countable family $\left\{\varphi_{k}\right\}$ in $X^{\prime}$ such that for all $B \in L_{\nu}(\mathscr{A})$, if $\left|\varphi_{k}(L(\nu))\right|(B)=0$ for all $k$, then $|\varphi(L(\nu))|(B)=0$ for all $\varphi$ normal and $\|\varphi\|_{n} \leq 1$.

Proof. The vector measure is countably additive, thus strongly additive. For the seminorm $\|\cdot\|_{n}$, we can prove that the family $\{|\varphi(L(\nu))|: \varphi$ normal and $\left.\|\varphi\|_{n} \leq 1\right\}$ of scalar measures is uniformly strongly additive by using the same proof as in [1, Proposition 17, page 8]. The uniform countable additivity follows from Lemma 2.4. Therefore, the lemma follows from Lemma 2.5 and the proof of the necessity part of Theorem 3.2.

Theorem 4.7. There exists a bounded, finitely additive internal positive measure $\mu$ on $(\Omega, \mathscr{A})$ with the following properties:

(1) For each $A \in \mathscr{A}, \mu(A) \approx 0$ if and only if $\left.\right|^{*} \varphi(\nu) \mid(A) \approx 0$ for all $\varphi \in X^{\prime}$.

(2) For each $B \in \sigma(\mathscr{A}), L(\mu)(B)=0$ if and only if

$$
\left|L\left(^{*} \varphi(\nu)\right)\right|(B)=0 \quad \text { for all } \varphi \in X^{\prime} .
$$

(3) $L_{\mu}(\mathscr{A})=L_{\nu}(\mathscr{A})=\bigcap_{\varphi \in X^{\prime}} L_{\varphi}(\mathscr{A})$ and, moreover, for each $B \in L_{\nu}(\mathscr{A})$, $L(\mu)(B)=0$ if and only if $|\varphi(L(\nu))|(B)=0$ for all $\varphi \in X^{\prime}$.

Proof. (1) By Lemmas 4.5 and 4.6, we can find a sequence $\left\{\varphi_{n}\right\}_{n \in \mathbb{N}}$ of normal continuous linear functionals such that for each $n,\left\|\varphi_{n}\right\|_{k} \leq 1$ for some $k$, and for any $B \in L_{\nu}(\mathscr{A})$, if $\left|\varphi_{n}(L(\nu))\right|(B)=0$ for every $n$, then $|\varphi(L(\nu))|(B)=0$ for all $\varphi \in X^{\prime}$. Consider the star extension $\left\{{ }^{*} \varphi_{n}\right\}_{n \in{ }^{*} \mathbb{N}}$ of $\left\{\varphi_{n}\right\}$. By the 
saturation property, there exists $\omega \in^{*} \mathbb{N}_{\infty}$ such that $\left.\left.\right|^{*} \varphi_{i}(\nu)\right) \mid(\Omega) \leq 1$ for all $i$ between 1 and $\omega$. As in $\S 3$, for each $A \in \mathscr{A}$, define

$$
\mu(A)=\left.\sum_{i=1}^{\omega}\right|^{*} \varphi_{i}(\nu) \mid(A) /\left(2^{i}\right) .
$$

It is easy to check that $\mu$ has the property stated in (1).

(2) It is clear that $\left.\varphi(L(\nu))\right|_{\sigma(\mathscr{A})}=\left.L\left(^{*} \varphi(\nu)\right)\right|_{\sigma(\mathscr{A})}$. Choose any $B \in \sigma(\mathscr{A})$ with $L(\mu)(B)=0$. Fix a positive integer $i$. For any given $\varepsilon>0, \exists A \in \mathscr{A}$ such that $A \supset B$ and $\mu(A)<\varepsilon / 2^{i}$. So, by the definition of $\mu$,

$$
\left.\frac{1}{2^{i}}\right|^{*} \varphi_{i}(\nu) \mid(A) \leq \mu(A)<\frac{\varepsilon}{2^{i}}, \quad \text { i.e., }\left.\right|^{*} \varphi_{i}(\nu) \mid(A)<\varepsilon .
$$

Thus, $\left|L\left(^{*} \varphi_{i}(\nu)\right)\right|(B)=0$ follows from the arbitrary choice of $\varepsilon$. By the property of the sequence $\left\{\varphi_{n}\right\}$, we know $\left|L\left(^{*} \varphi(\nu)\right)\right|(B)=|\varphi(L(\nu))|(B)=0$ for all $\varphi \in X^{\prime}$. For the other part of (2), we assume $\left|L\left({ }^{*} \varphi(\nu)\right)\right|(B)=0$ for all $\varphi \in X^{\prime}$. For any given $\varepsilon>0$, choose a positive integer $m$ such that $\frac{1}{2^{m}}<\frac{\varepsilon}{2}$. Since $\left|L\left({ }^{*} \varphi_{i}(\nu)\right)\right|(B)=0$ for $1 \leq i \leq m, \exists A \in \mathscr{A}$ such that $B \subset A$ and $\left.\right|^{*} \varphi_{i}(\nu) \mid(A)<\frac{\varepsilon}{2}$. So $\mu(A) \leq \frac{\varepsilon}{2}+\frac{1}{2^{m}}<\varepsilon$. Thus, $L(\mu)(B) \leq \varepsilon$. Therefore $L(\mu)(B)=0$.

(3) For any $B \in L_{\mu}(\mathscr{A}), \exists A_{1}, A_{2} \in \sigma(\mathscr{A})$ and $B^{\prime} \subset A_{2}$ such that $A_{1} \cap A_{2}$ $=\varnothing, B=A_{1} \cup B^{\prime}$, and $L(\mu)\left(A_{2}\right)=0$. By the result in (2), we know $\left|L\left({ }^{*} \varphi(\nu)\right)\right|\left(A_{2}\right)=0$ for all $\varphi \in X^{\prime}$. Thus, $B^{\prime} \in L_{\varphi}(\mathscr{A})$ and $\left|L\left(^{*} \varphi(\nu)\right)\right|\left(B^{\prime}\right)=0$. By choosing the empty set $\varnothing$ as a $\nu$-approximation of $B^{\prime}$, we have $B^{\prime} \in$ $L_{\nu}(\mathscr{A})$. Whence $B \in L_{\nu}(\mathscr{A}) \subset \bigcap_{\varphi \in X^{\prime}} L_{\varphi}(\mathscr{A})$. Therefore

$$
L_{\mu}(\mathscr{A}) \subset L_{\nu}(\mathscr{A}) \subset \bigcap_{\varphi \in X^{\prime}} L_{\varphi}(\mathscr{A}) \subset \bigcap_{n=1}^{\infty} L_{\varphi_{n}}(\mathscr{A}) .
$$

On the other hand, for any $B \in \bigcap_{n=1}^{\infty} L_{\varphi_{n}}(\mathscr{A})$ and given $\varepsilon>0$, there exists $m \in \mathbb{N}$ such that $\frac{1}{2^{m}}<\frac{\varepsilon}{2}$. Since $B \in \bigcap_{n=1}^{m_{n}} L_{\varphi_{n}}(\mathscr{A})$, we can find $A_{1}, A_{2} \in \mathscr{A}$ such that $A_{1} \subset B \subset A_{2}$ and $\left.\right|^{*} \varphi_{n}(\nu) \mid\left(A_{2}-A_{1}\right)<\frac{\varepsilon}{2}$ for all $1 \leq n \leq m$. So $\mu\left(A_{2}-A_{1}\right)<\frac{\varepsilon}{2}+\frac{1}{2^{m}}<\varepsilon$. Thus $B \in L_{\mu}(\mathscr{A})$. Hence $\bigcap_{n=1}^{\infty} L_{\varphi_{n}}(\mathscr{A}) \subset L_{\mu}(\mathscr{A})$. Therefore

$$
L_{\mu}(\mathscr{A})=L_{\nu}(\mathscr{A})=\bigcap_{\varphi \in X^{\prime}}^{\infty} L_{\varphi}(\mathscr{A})=\bigcap_{n=1}^{\infty} L_{\varphi_{n}}(\mathscr{A}) .
$$

It is clear that the rest of (3) follows easily from the previous proof.

\section{ACKNOWLEDGMENTS}

The authors are indebted to Professor Peter Loeb for his very valuable help. They are also grateful to Professors Ward Henson and Jerry Uhl for helpful discussions. The second author's research has been supported in part by University of Illinois Fellowships and by the National Science Foundation of America under contracts DMS-8702064 and DMS-8902095. 


\section{REFERENCES}

1. J. Diestel and J. J. Uhl, Vector measures, Mathematical Surveys Number 15, Amer. Math. Soc., Providence, RI, 1977.

2. A. Grothendieck, Sur les applications linearies faiblement compactes d'espaces du type $C(K)$, Canad. J. Math. 5 (1953), 129-173.

3. C. W. Henson and L. C. Moore, Nonstandard analysis and theory of Banach spaces, Nonstandard analysis-recent developments (A. E. Hurd, ed.), Lecture Notes in Math., vol. 983, Springer-Verlag, Berlin, 1983.

4. A. E. Hurd and P. A. Loeb, An introduction to nonstandard real analysis, Academic Press, Orlando, Florida, 1985.

5. P. A. Loeb, Conversion from nonstandard to standard measure spaces and applications in probability theory, Trans. Amer. Math. Soc. 211 (1975), 113-122.

6. _ A functional approach to nonstandard measure theory, Contemp. Math. 26 (1984), 251-261.

7. P. A. Loeb and H. Osswald, Nonstandard integration theory in solid Riesz space (to appear).

8. Y. Sun, On the theory of vector valued Loeb measures and integration (to appear).

9. $\ldots$ A nonstandard proof of the Riesz representation theorem for weakly compact operators on $C(\Omega)$, Math. Proc. Comb. Phil. Soc. 105 (1989), 141-145.

10. _ A Banach space in which a ball is contained in the range of some countably additive measure is superreflexive, Canad. Math. Bull. 33 (1990), 45-49.

11. _ Nonstandard theory of vector measures, Ph.D. dissertation, University of Illinois, Urbana, Illinois, 1989.

12. R. T. Zivaljevic, Loeb completion of internal vector valued measures, Math. Scand. $\mathbf{5 6}$ (1985), 276-286.

Mathematisches Institut, Universität MÜNChen, Theresienstrasse 39, D 8000 MÜNCheN 2, West GeRMANY

Department of Mathematics, University of Illinois, Urbana, IllinoIs 61801

Current address: Department of Mathematics, National University of Singapore, Singapore 0511, Republic of Singapore 